\title{
Technik für Senioren - ein Zukunftsmarkł für Brandenburger Unternehmen
}

\author{
Ausgewählte Ergebnisse eines Forschungsprojekts ${ }^{1}$
}

\author{
Rainer Voß, Martina Brandt
}

\section{Technologieentwicklung und Markt- erschließung für den Strukturwandel}

Im Land Brandenburg sind seit Jahren die Anstrengungen darauf gerichtet, mit verschiedenen wirtschafts-, technologie- und regionalpolitischen Strategien den Strukturwandel in der Wirtschaft mit ihren fast ausschließlich kleinen und mittleren Unternehmen zu bewältigen. Eine strategische Richtung basiert auf der Technologiekonzeption des Landes Brandenburg ${ }^{2}$ und ist auf die schwerpunktmäßige Förderung ausgewählter Technologiefelder gerichtet. Dabei spielen Aus- und Neugründungen kleinster und kleiner Unternehmen eine wichtige Rolle. Ein Beispiel für unïbersehbare Erfolge dieser Strategie in den zurïckliegenden Jahren ist zweifellos die Biotechnologie. Hier ist es gelungen, an verschiedenen Standorten sowohl im Berliner Umland als auch in der strukturschwachen entfernteren Region Luckenwalde „Nuklei“ aus der Verbindung von Wissenschaft und Unternehmen in Form von Biotechnologiezentren mit ersten wirtschaftsstrukturellen Effekten zu schaffen. ${ }^{3}$

Eine zweite strategische Richtung besteht in der an vorhandene Branchenpotentiale anknüpfenden Wirtschaftsförderung, die durch Verbundaktivitäten, die Bildung strategischer Allianzen und Innovationsnetzwerke unterstuitzt wird. Beispiele hierfür sind die Bereiche Verkehrstechnik, Nachwachsende Rohstoffe, Tourismus, Papier- und Druckindustrie, Textilgewerbe und Mode. ${ }^{4}$ Hier geht es darum, den Zugang Brandenburger Unternehmen zu aussichtsreichen Märkten zu erschließen und vorrangig die technologischen Kernkompetenzen der Unternehmen dafür zu entwickeln.

Als Zukunftsmärkte werden weltweit auch die mit dem wachsenden Anteil älterer Menschen an der Gesamtbevölkerung verbundenen gesellschaftlichen und individuellen Problemlagen bewertet. Dies gilt sowohl für die Nachfrage als auch für die erst durch Forschung und Entwicklung hervorzubringenden und dann durch Unternehmen zu vermarktenden innovativen Leistungsangebote. Jene betreffen solche in sich stark differenzierten Gebiete wie Medizintechnik, Gesundheits- und Rehatechnik, Pflegetechnik, Haushaltstechnik, Kommunikationstechnik, Transporttechnik, Wohnungsanpassung und Sicherheitstechnik.

Nachfrageumfang und -tempo dieser zukünftigen Märkte lassen sich derzeit allerdings nur schwer qualitativ und quantitativ differenziert abschätzen. Sie werden sich in den nächsten Jahren z.B. durch sich weiter wandelnde Lebensstile, verändertes Konsum- und Kaufverhalten älterer Menschen, neue Leistungsangebote, wachsende bzw. zusätzlich verfuigbare Einkommen und sich verändernde gesellschaftliche Rahmenbedingungen erst herausbilden. Nur in wenigen Bereichen - vor allem in der Medizin- und Rehatechnik - kann man von bereits fest etablierten Märkten hinsichtlich Nachfrage und Angebot sprechen.

Einige Regionen in Deutschland (z.B. Nordrhein-Westfalen und Sachsen) verstärken in jüngster Zeit ihre Anstrengungen zur „konzertierten“ Erschließung erwarteter Marktpotentiale. ${ }^{5}$

\section{Projekthintergrund und Zielsetzung}

Angesichts dieser Entwicklungen stellt sich die Frage, ob der Bereich „Technik für Senioren und altersgerechtes Wohnen" auch für Brandenburger Unternehmen Perspektiven eröffnet und hiervon langfristig wirtschaftsstrukturelle Effekte für die Region erwartet werden können.

Die Technologie- und Innovationsagentur Brandenburg (T.IN.A.) hat deshalb mit Bezug auf die Förderung im Rahmen der Gemeinschaftsinitiative RECHAR II der Europäischen Kommission ${ }^{6}$ für die RECHAR-Gebiete des Landes (Stadt Cottbus, Landkreise Spree-Neiße, ElbeElster, Oberspreewald-Lausitz) ein Projekt initiiert, das für diesen Bereich die Schaffung eines Transferzentrums anstrebt. Durch die Tätigkeit des Zentrums sollen die wirtschaftlichen Aktivitäten kleiner Unternehmen auf dem Gebiet „Seniorengerechte Technik“, ihre Kooperationen mit Forschungseinrichtungen sowie eine Zusammenarbeit in Unternehmensverbünden initiiert und nachhaltig unterstuitzt werden. Im Rahmen einer Machbarkeitsstudie erarbeitete ein Projektteam - bestehend aus Fachleuten verschiedener Einrichtungen aus Wissenschaft und Wirtschaft - die fuir die Investitionsentscheidung notwendigen Grundlagen. ${ }^{7}$

Die Thematik „Altern und Technische Hilfen“ wurde in gesellschafts-, sozial- und gesundheitspolitischen Studien und Forschungsprojekten in den letzten 10-15 Jahren zum Teil sehr tiefgehend und differenziert analysiert. Allerdings spielten marktbezogene Fragestellungen, z.B. 
zur betriebswirtschaftlich relevanten Nachfrage und zu Kompetenzprofilen kleiner und mittlerer Unternehmen sowie zu wirtschaftsstrukturellen Effekten bisher kaum eine Rolle. Da es sich jedoch bei Technischen Hilfen um innovative Produkte handelt, die mit komplizierten Nachfrageprozessen verbunden sind und mit technologischer Kompetenz, nachfrageorientiert und wettbewerbsfähig von Unternehmen zu entwickeln, herzustellen und zu vertreiben sind, standen im Teilprojekt der Autoren im Rahmen der o.g. Machbarkeitsstudie folgende Fragen im Mittelpunkt der Untersuchung:

1. Verfügen diese Märkte uiber genuigend quantitatives und qualitatives Nachfragepotential, um einer größeren und wachsenden Zahl von Anbietern, einschließlich möglicher „Newcomer“ Differenzierungsoptionen für innovative Leistungsangebote zu eröffnen?
2. Wie sieht die derzeitige Angebotssituation aus, welche Anbieterstrukturen und -strategien bilden sich heraus?

3. Welche Unternehmen in der Region sind hier bereits aktiv bzw. haben begründetes Interesse an Geschäftsfeldern im Bereich Technische Lebenshilfen?

4. Welche Zutrittschancen haben solche Unternehmen zu diesen Märkten, von welchen unternehmensinternen und -externen Ressourcen und Bedingungen hängen diese Chancen $\mathrm{ab}$ ?

Während die Analyse des Nachfragepotentials vorwiegend als Sekundäranalyse unter Nutzung verfügbarer soziologischer, sozialstatistischer, techniksoziologischer, gesundheits- und rehabilitationswissenschaft-

\section{PROBLEMSITUATION}

Altern ist begleitet von personen- und umweltbedingten Veränderungen, die neue Anforderungen an ein selbstbestimm tes Leben, eine selbständige Haushaltsführung in der eigenen Wohnung, die soziale Integration älterer Menschen und deren Teilhhabe am gesellschaftlichen Leben stellen.

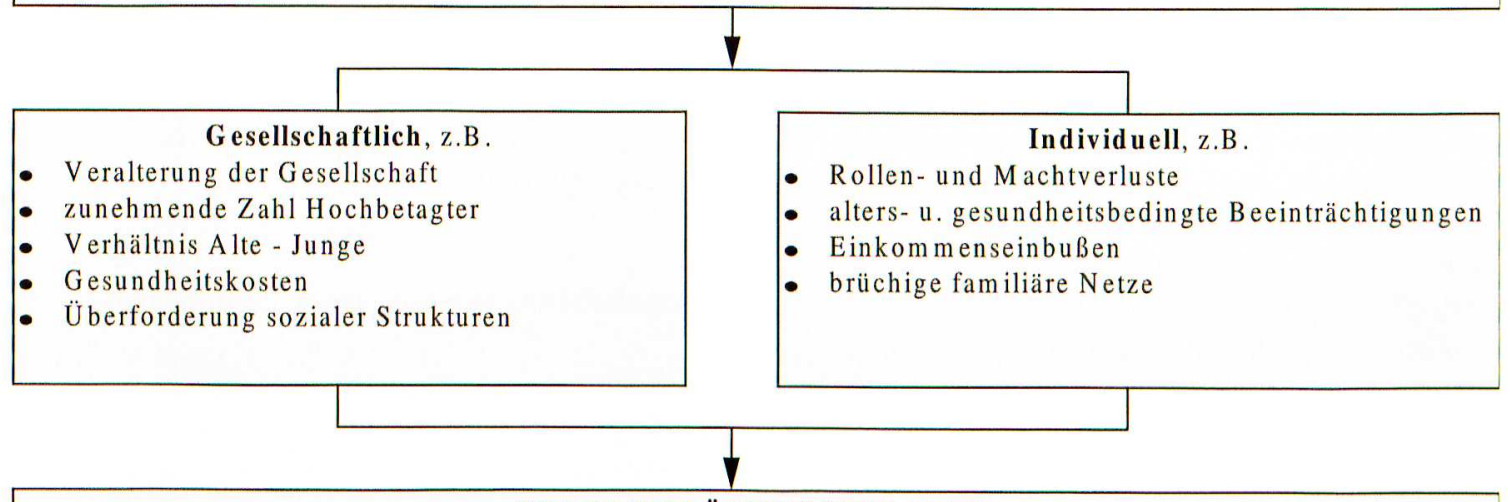

\section{PROBLEMLÖSUNGSBEDARFE}

Effiziente Kompensation von Verlusten an Kompetenz zur Bewältigung von Umweltanforderungen zum Erhalt einer selbstbestimmten und selbständigen Lebensführung und zur Sicherung einer zufriedenstellenden Lebensqualität im Alter

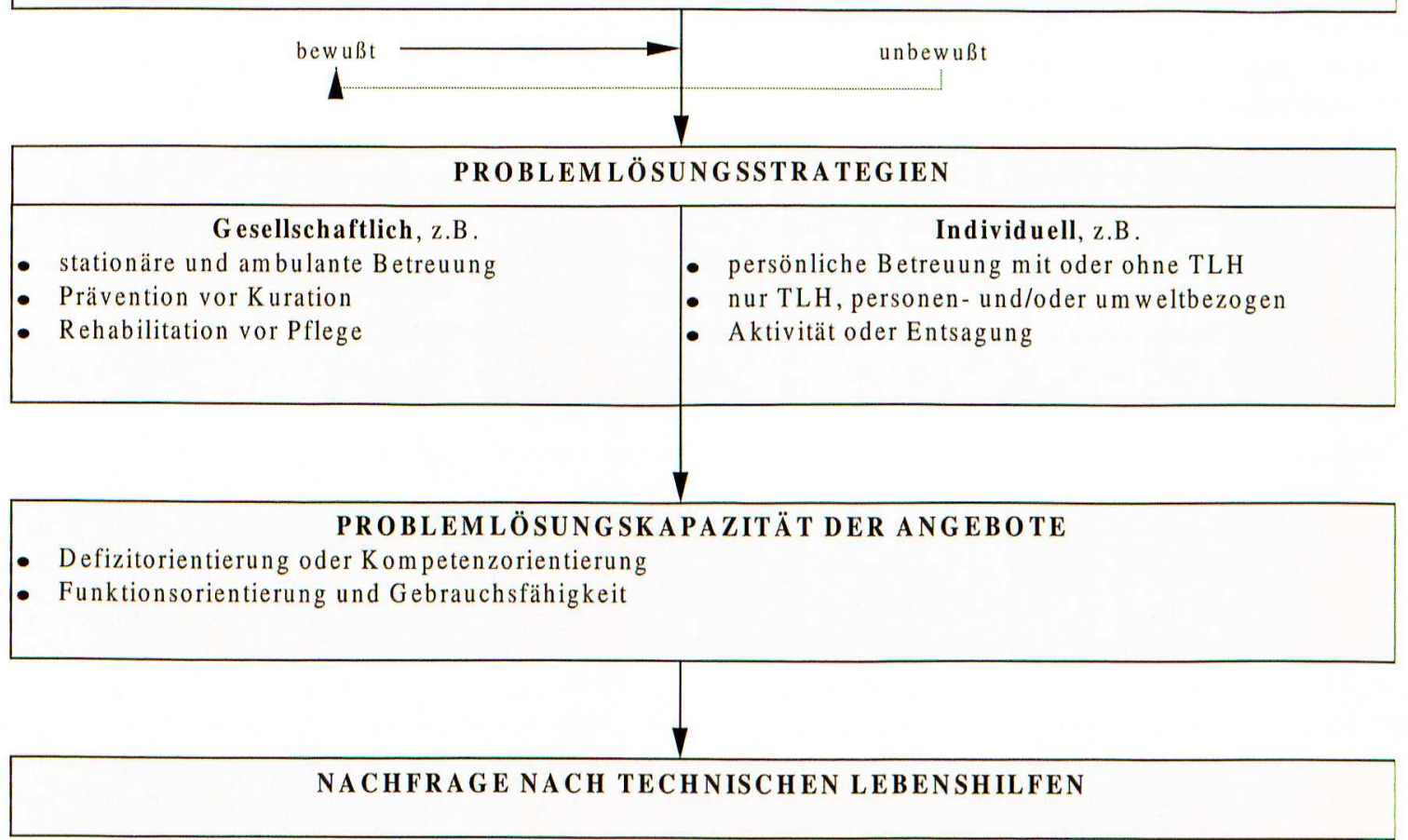


licher Quellen erfolgte, basiert die Bewertung des Angebotspotentials wesentlich auf den Ergebnissen einer im Rahmen des Projekts durchgeführten schriftlichen Unternehmensbefragung. Sowohl für die Untersuchung der Nachfrage als auch des Angebotspotentials wurden auch moderierte Gruppendiskussionen mit relevanten Akteursgruppen im Rahmen von Workshops durchgefuihrt. Sie dienten sowohl der Beschaffung regionalspezifischer Informationen zur Nachfrage- und Anbietersituation als auch der Verifizierung der aus der Unternehmensbefragung für das zu schaffende Tranferzentrum abgeleiteten Schlußfolgerungen.

\section{Analyse und Bewertung des Nachfrage- potentials}

\subsection{Zusammenhang zwischen Problem- situationen und Nachfrageentwicklung}

Die Nachfrage nach technischen Produkten kann als Ausdruck eines Problemlösungsbedarfs verstanden werden, für dessen Befriedigung Menschen Lösungsangebote suchen. Dieser Problemlösungsbedarf ist das Resultat einer bestimmten Problemsituation (vgl. Abb.1) und stellt sich für den Bereich Technische Lebenshilfen sehr differenziert dar:

Die Älteren als Nachfrager nach Technischen Lebenshilfen stellen keine homogene Bevölkerungsgruppe dar. Aus der großen Spannweite ihrer Lebensstile resultieren höchst unterschiedliche Ansprüche an ein selbstbestimmtes Leben, deren Umsetzung mittels technischer Hilfen (nach auf Art und Umfang) sowohl von sozial determinierten als auch von individuellen Faktoren abhängt.

Etwa 20 Prozent der älteren Menschen sind hilfsbeduirftig (aufgrund altersbedingter Beeinträchtigungen oder krankheitsbedingter Hilfebedürftigkeit bis hin zu dauerhafter Behinderung), 80 Prozent der älteren Bürger leben dagegen bis ins hohe Lebenshalter hinein relativ gesund, selbstbestimmt und selbständig in ihrer gewohnten häuslichen Umgebung bei aktiver gesellschaftlicher Teilhabe. ${ }^{8}$ Allerdings wird dies gegenwärtig nicht mit exakten Nachfrageabschätzungen, z.B. im Bereich von Freizeit, Haushaltsführung, Mobilität und Kleidung belegt. Nur etwa 10 Prozent der älteren Menschen werden beim heutigen Stand der Medizin pflegebedürftig, wovon drei Viertel von Angehörigen und ambulanten Hilfsdiensten zu Hause versorgt werden. ${ }^{9}$

Daraus resultieren unterschiedliche Nachfragesituationen z.B. für Alltagstechnik, die Tätigkeiten jedes älteren (möglichst auch jüngerer) Menschen erleichtert oder gar erst ermöglicht und für Technische Hilfen, die zur Kompensation gesundheitlicher Beeinträchtigungen oder gar von Behinderungen entwickelt werden müssen.

\subsection{Nachfragebeeinflussende Faktoren}

Wenn man die in der Literatur enthaltenen Informationen zur Problematik „Altern und Gesellschaft" analysiert, die z.B. in Zusammenhängen mit Wohlfahrtsuntersuchungen, Sozialstrukturanalysen, Lebensstilen und Selbstbestimmtheit im Alter sowie mit der Hilfsbedürftigkeit älterer Menschen erzeugt und verwendet wurden, lassen sich hinsichtlich ihrer Relevanz für die Entwicklung von Nachfrage nach seniorengerechter Technik folgende Einflußfaktoren begruinden:

\section{Demografische Entwicklung}

Während vor 50 Jahren lediglich 7 Prozent der Bevölkerung der EU älter als 60 Jahre waren, werden es im Jahre 2020 bereits 25 Prozent sein. ${ }^{10}$ Für Brandenburg wird eine Zunahme der älteren Bevölkerung bis 2015 um fast 40 Prozent prognostiziert. Hervorzuheben ist die geschlechtsspezifische Ungleichgewichtung bei den Älteren über 65 Jahre. In dieser Altersgruppe beträgt der Frauenanteil in Brandenburg 65 Prozent, in jener der über 80jährigen sogar 75,7 Prozent. ${ }^{11}$

\section{Einkommensverhältnisse}

Die für Deutschland insgesamt sehr positive Einschätzung der Vermögenslage der über 60jährigen (1,5 Billionen DM Vermögen im Jahre 2000) ${ }^{12}$ läßt sich allerdings nicht uneingeschränkt auf Brandenburg übertragen, da in den neuen Bundesländern das Alterseinkommen zu 98 Prozent aus der gesetzlichen Altersrentenversicherung resultiert und die ältere Generation hier ein unvergleichbar geringeres Vermögen besitzt. ${ }^{13}$ Diese Ost-West-Unterschiede werden sich jedoch mit dem Nachrücken künftiger Altengenerationen immer weiter angleichen.

\section{Morbidität und Behinderung}

Technische Hilfen für Tätigkeiten mit hohen Anforderungen an Muskelkraft, Bewegungsflexibilität und die Leistungsfähigkeit anderer Körperfunktionen treffen im höheren Lebensalter auf eine Nachfrage, da diese eine deutliche Tendenz der Verschlechterung aufweisen (Unausweichlichkeit des körperlichen Verfalls ab 85). Allerdings spricht einiges dafür, daß die Zahl der Pflegebedürftigen nicht unbedingt deutlich zunimmt, eher wird sich der Schweregrad verändern. ${ }^{14}$ Langfristige Pflegebedürftigkeit als neues soziales Risiko wird deshalb als ernstzunehmendes Problem in allen europäischen Ländern diskutiert. Hier sind ebenfalls Auswirkungen auf die Art der Nachfrage nach Technischen Hilfen zu erwarten.

Technische Hilfen für Schwerbehinderte (in Deutschland 6,5 Millionen Menschen mit mindestens 50 Prozent Behinderung) müssen zum überwiegenden Teil auf ältere Menschen zugeschnitten sein, da über die Hälfte älter als 65 ist (in Brandenburg waren es Ende 1996 sogar 60 Prozent) und weitere 24,8 Prozent der Altersgruppe 55-65 Jahre angehören. ${ }^{15}$ 


\section{Wohnsituation und Technikausstattung}

Da ältere Menschen nahezu 80 Prozent der Zeit in ihrer Wohnung verbringen und 95 Prozent der heute uiber 65jährigen in nicht angepaßten Wohnungen leben, sind seniorengerechte bauliche und technische Ausstattungen fuir den Bereich Wohnen von besonderer Relevanz. ${ }^{16}$ In den neuen Bundesländern ist die Wohnsituation Älterer durch überproportional hohe Anteile an schlecht ausgestatteten Wohnungen geprägt. Rund 22 Prozent der älteren Menschen leben in Brandenburg in Wohnungen mit extremem Substandard (ohne InnenWC). Auch knapp ein Viertel der Wohnungen/Häuser, die von älteren Eigentuimern selbst genutzt werden, weist diese schlechte Qualität auf. ${ }^{17}$ Befragungen einzelner Kommunen (z.B. in Zeuthen) ${ }^{18}$ nach Veränderungswünschen beziehen sich deshalb vor allem auf bauliche Verbesserungen im Sanitärbereich sowie auf die Beseitigung von Türschwellen.

Für die Nachfrage nach Mobilitätshilfen außerhalb der Wohnung könnte sich die Tatsache fördernd auswirken, daß in den neuen Bundesländern lediglich zwei Fünftel der Älteren ab 55 Jahre über einen privaten PKW verfügen. Von den 60jährigen und älteren Alleinlebenden haben sogar nur drei Prozent ein Auto zur Verfügung. ${ }^{19}$

Auch die in Brandenburg vorhandenen ca. 65 Anbieter für Fahr- und Begleitdienste, die die Mobilität Behinderter gewährleisten helfen, decken keinesfalls die Nachfrage. Insgesamt steht der Aufbau einer behindertengerechten Infrastruktur, angefangen von der Straßenraumgestaltung bis hin zu Übernachtungsmöglichkeiten auf Reisen, erst am Anfang.

\section{Einstellungen und Technikakzeptanz}

Aufgrund einer zunehmenden Vertrautheit künftiger Altengenerationen mit Technik kann in den nächsten Jahren mit einem Ansteigen der Akzeptanzbereitschaft der Älteren gegenüber Technischen Lebenshilfen gerechnet werden. Etwa 25 Prozent der älteren Generation haben bereits den Wertewandel vom konservativfamilienorientierten Senior hin zum „genuß- und konsumfreudigen Alten" vollzogen.

Es haben jedoch nur Produkte eine Chance, die nicht stigmatisieren, d.h., die die Käufer nicht per se als Senioren ansprechen. Generell scheint bei Älteren die Neuerungsbereitschaft auf der Einstellungsebene höher als auf der Handlungsebene zu sein. Qualität ist ein wichtiges Kaufkriterium und hat bei der Kaufentscheidung einen höheren Stellenwert als die Preiswürdigkeit. Senioren erwarten beim Kauf einen spürbaren Nutzen und wollen beraten werden, benötigen mehr Zeit für die Kaufentscheidung und haben eine eigene Produktphilosophie. ${ }^{20}$

\section{Bedingungen für Selbsthilfe im Alter}

Die derzeitigen Bedingungen für Selbsthilfe im Alter sind in allen europäischen Ländern mehr oder weniger ungünstig und auch die diesbezüglich zu erwartenden Trends lassen einen zunehmenden Bedarf nach Technischen Hilfen im Alter erwarten. So wächst der Anteil alleinlebender Hilfe- und Pflegebeduirftiger ständig an. Gleichzeitig geht die familiäre Pflegebereitschaft und -möglichkeit aufgrund fehlender Angehöriger bzw. deren räumlicher Entfernung zurüick. In Brandenburg kommt hinzu, daß die durchschnittliche Kinderzahl in der Gruppe der 65-70jährigen mit 2,0 vergleichsweise geringer ist als die der anderen Altersgruppen und der Anteil alleinlebender älterer Frauen besonders hoch ist. ${ }^{21}$

\section{Qualität und Struktur des derzeitigen Versorgungs- systems}

Nur für den relativ kleinen Teil der medizinisch induzierten Technischen Hilfen übernehmen die Kassen die Finanzierung. Hilfsmittel, die die Pflege und Betreuung erleichtern sowie die Umgestaltung der häuslichen Wohnumgebung und altengerechte Geräte der Alltagstechnik werden - wenn überhaupt - nur uiber die Sozialämter finanziert.

Die Bedingungen dafür, daß den Bedürftigen diese Technischen Hilfen tatsächlich zukommen, sind in Deutschland derzeit unguinstig (keine systematische und gezielte Information, wenige ausgebildete Gerontologen, komplizierte Bestimmungen des Bundessozialhilfegesetzes und der Krankenkassen, z.T. nicht eindeutig geregelte Ansprüche, Mangel an Koordinationsmöglichkeiten) und lassen einen Bedarf an Beratungsstellen erkennen. Für Brandenburg und speziell die RECHARRegion dürften besondere Anforderungen an die Formen der Beratungsangebote aus der vorhandenen geringen Bevölkerungsdichte resultieren.

Auf einem Workshop mit Vertretern von sozialen Einrichtungen, Senioren- und Behindertenverbänden in Cottbus im Rahmen der Projektbearbeitung wurden die Probleme bei der Versorgung mit Technischen Lebenshilfen, Alltagstechnik und Wohnraumanpassungsmaßnahmen auch für die Region deutlich. Hervorgehoben wurde insbesondere das Informationsproblem bei der Beantragung, Finanzierung und Anpassung. Zugleich schätzten die Teilnehmer für verschiedene Bereiche Technischer Hilfen (z.B. zur Unterstuitzung von Pflegeleistungen, zur Erhaltung der Kommunikationsfähigkeit und der Mobilität, zur Gestaltung freier Zeit und Überbrückung des Alleinseins) einen zwar gegenwärtig unterschiedlichen, jedoch durchweg zukünftig wachsenden Nachfrageumfang ein.

\section{Derzeitiges Versorgungsniveau Technischer Hilfen}

Technische Hilfen für beeinträchtigte und behinderte Menschen sind in großer Vielfalt und Differenziertheit verfügbar (geschätzt werden in Deutschland rund 10.000 bis 12.000 Hilfsmittel). Die uiber Wohlfahrtsverbände und Sozialstationen mediatisierte Beziehung zwischen Hersteller und Kunden bewirkt jedoch eine eingeschränkte Markttransparenz. Charakteristisch für das derzeitige Versorgungsniveau ist deshalb, daß die erworbenen Technischen Hilfen von den Betroffenen 
häufig nur selten oder gar nicht genutzt werden (ca. jedes 7. bis 8. angeschaffte Hilfsmittel stellt eine Fehlversorgung dar). Stark kritisiert werden häufig auch die Qualität, der Preis und das Design der Produkte. Viel zu stark steht bei den Herstellern wie bei der Versorgung die Funktionalität und viel zu wenig die Gebrauchsfähigkeit der Hilfsmittel im Vordergrund. ${ }^{22}$ Die stärkere Berüicksichtigung der Gebrauchsfähigkeit setzt allerdings voraus, über Forschungsaktivitäten und angepaßte Marketingmaßnahmen die differenzierten Anforderungen von Senioren an Technische Hilfen bei Beeinträchtigungen und im Alltag sowie bei Wohnungsanpassungen differenzierter zu erfassen und in innovative Leistungsangebote umzusetzen.

\subsection{Zusammenhänge zwischen den Einflußfaktoren}

Inwieweit sich die oben dargestellten Entwicklungen direkt oder vermittelt fördernd bzw. hemmend auf die Nachfrage nach konkreten Leistungsangeboten auswirken, kann aus mehreren Gründen nur auf der Basis tiefergehender Analysen abgeschätzt werden.

Erstens unterliegt das Verhalten zu Technischen Hilfen sehr stark dem Einfluß individueller Faktoren, die für einzelne Anwendungsbereiche völlig unterschiedlich ausgeprägt sind. Zweitens können Vermittler wie Ärzte, Ergotherapeuten, soziale Dienste, Sanitätshäuser etc. die derzeit vorhandenen Luicken in der Wissensbasis schließen, was nachfrageauslösend bzw. -verstärkend wirken kann. Drittens muß beachtet werden, daß die Entwicklung der o.g. Prozesse und Faktoren sich selbst wechselseitig beeinflußt und darüber hinaus dem Einfluß weiterer Größen (z.B. Maßnahmen im Rahmen der Arbeitsmarkt-, Wohnungsbau- oder Sozialpolitik) unterliegt. Inwieweit sich hier Einflußfaktoren in ihrer Nachfragewirkung verstärken, überlagern oder auch gegenläufig verhalten bzw. aufheben, kann erst durch eine auf Vernetzung basierende Analyse transparent gemacht werden. Als Voraussetzung hierzu miißten im Rahmen weiterfuihrender Analysen die einzelnen nachfragebeeinflussenden Faktoren auf konkrete Bereiche Technischer Lebenshilfen bzw. konkrete Produktgruppen oder sogar einzelne Innovationen bezogen analysiert werden.

\section{Angebotssituation und Anbieterstruk- turen im Markt für seniorengerechte Technik in Deutschland}

Bundesweit sind über 1000 Unternehmen auf dem Gebiet Technische Lebenshilfen tätig. Eine 1995 veröffentlichte Befragung von 956 Anbietern im gesamten Bundesgebiet mit einer Rücklaufquote von 25 Prozent erbrachte erstmalig anhand der Angaben von über 200 Firmen aussagekräftige Ergebnisse zur Struktur und Merkmalen deutscher Anbieter von Senioren- und Behindertentechnik. ${ }^{23}$ Danach sind 75 Prozent der Unternehmen bereits seit 1980 auf diesem Gebiet tätig. Nur reichlich ein Drittel (37 Prozent) sind ausschließlich auf dem Altenmarkt aktiv. Aus der Tatsache, daß zwei Drittel dieser
Unternehmen ihre größte Umsatzsteigerung in den Jahren 91 bis 93 zu verzeichnen haben, läßt sich ableiten, daß die Nachfrageentwicklung in diesen sich aufbauenden Märkten ungleichmäßig und zum Teil sprunghaft verläuft und Unternehmen demzufolge in der Lage sein müssen, längere Durststrecken zu uiberbrücken.

Der Anbietermarkt für Alltagstechnik für Senioren ist noch stark unterentwickelt. In Deutschland werden nur 0,4 Prozent des Umsatzes mit angepaßten Leistungen für die Haushaltsfuihrung erzielt, demgegenüber aber 30 Prozent fuir Leistungsangebote zur Mobilitätserhöhung. Insbesondere auf dem Haushaltsgerätesektor und in der Kommunikationstechnik stehen kleine Unternehmen mit ihren Innovationen großen, leistungsstarken Unternehmen gegenüber, denen die Rolle des „Seniorenmarktes“ ebenfalls zunehmend bewußt wird.

\subsection{Kernkompetenzen bereits aktiver und weiterer interessierter Firmen in der Region}

Eine überwiegend in Südbrandenburg durchgeführte schriftliche Befragung von 237 Firmen mit einem Rücklauf von 34 Prozent (79 Unternehmen) ergab, daß hier bereits 46 Unternehmen auf dem Gebiet „Gesundheitstechnik, Technische Lebenshilfen, altersgerechtes Wohnen" tätig sind.

Die Firmen produzieren nicht ausschließlich für diesen Markt, sondern sind in der Regel nur mit 1 bis 2 Einzelprodukten und weniger als Anbieter von System- oder Komplettlösungen präsent. Dies war auch ein Ergebnis der gesamtdeutschen Analyse. Das Angebotsspektrum ist dabei insgesamt sehr vielfältig und umfaßt z.B. Sensorsysteme, Lichtruf- und Personensuchanlagen, Therapiegeräte, Geräte und Instrumente für minimalinvasive Chirurgie, Hard- und Softwarelösungen für Pflegedienste und Heime, altersgerechte Badewannen, behindertengerechte Anpassung von Straßenbahnwagen, Gelenkimplantate oder Sanitär- und Hilfseinrichtungen für barrierefreies Wohnen.

Die Mehrheit der gesunden und aktiven älteren Menschen (die sogenannten „neuen Alten“) spielt allerdings in der Leistungsorientierung der Firmen bislang kaum eine Rolle. Jedoch produzieren die meisten entsprechend ihres auf Medizin-, Behinderten- sowie REHATechnik ausgerichteten Profils für alle betroffenen Altersgruppen und nicht spezifisch angepaßt nur für ältere Menschen - eine Tatsache, die im Hinblick auf eine mögliche Hinwendung zur Alltagstechnik wiederum als vorteilhaft einzuschätzen ist.

Zu den 46 bereits auf dem Gebiet Technische Lebenshilfen aktiven Firmen kommen weitere 24 befragte Firmen hinzu, die ihr Interesse erklärt haben, sich auf solche Geschäftsfelder zu orientieren sowie zusätzlich über 20 Unternehmen, die sich zwar nicht an der Befragung beteiligten, aber an dem im Rahmen der Projektbearbeitung durchgefuihrten Unternehmerworkshop teilnahmen. Somit kann man von insgesamt 90 Unternehmen - vorwie- 
gend in Südbrandenburg ansässig - ausgehen, die am Feld Technische Lebenshilfen interessiert sind.

Die meisten dieser Unternehmen verfuigen über zum Teil sehr differenzierte technologische Kernkompetenzen, die eine Basis für eine verstärkte bzw. erstmalige Orientierung auf seniorengerechte Technik bilden könnten. Das betrifft solche Bereiche wie Sensorik, Automatisierung, minimalinvasive Medizin, Vakuumtechnologien, Kunststofftechnologien, Metallbe- und -verarbeitung, ökologisches Bauen, Signalerfassung und -verarbeitung, MSR-Technologien, Softwaretechnologien bis hin zu Kybernetik und Bionik.

Bezüglich solcher Unternehmensmerkmale wie Beschäftigtenzahl (vgl. Abb. 2) und Umsatz (vgl. Abb. 3) handelt es sich überwiegend um kleinste und kleine Unternehmen, die sich damit kaum von den Unternehmen unterscheiden, die im Rahmen der gesamtdeutschen Befragung erfaßt wurden.

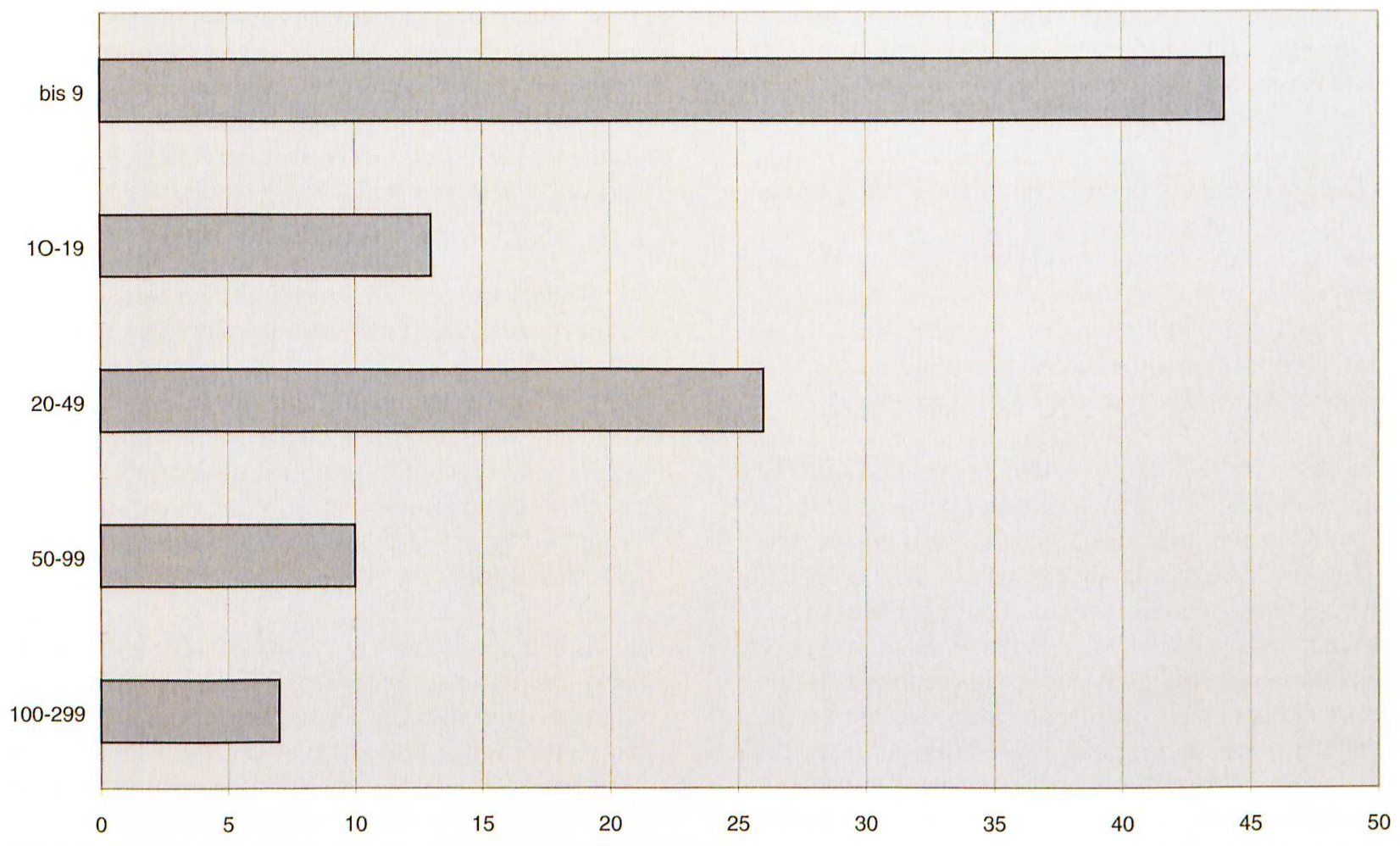

Abb. 2: Betriebsgröße Brandenburger Unternehmen (Mitarbeiter) ( $n=70$, Angaben in \%)

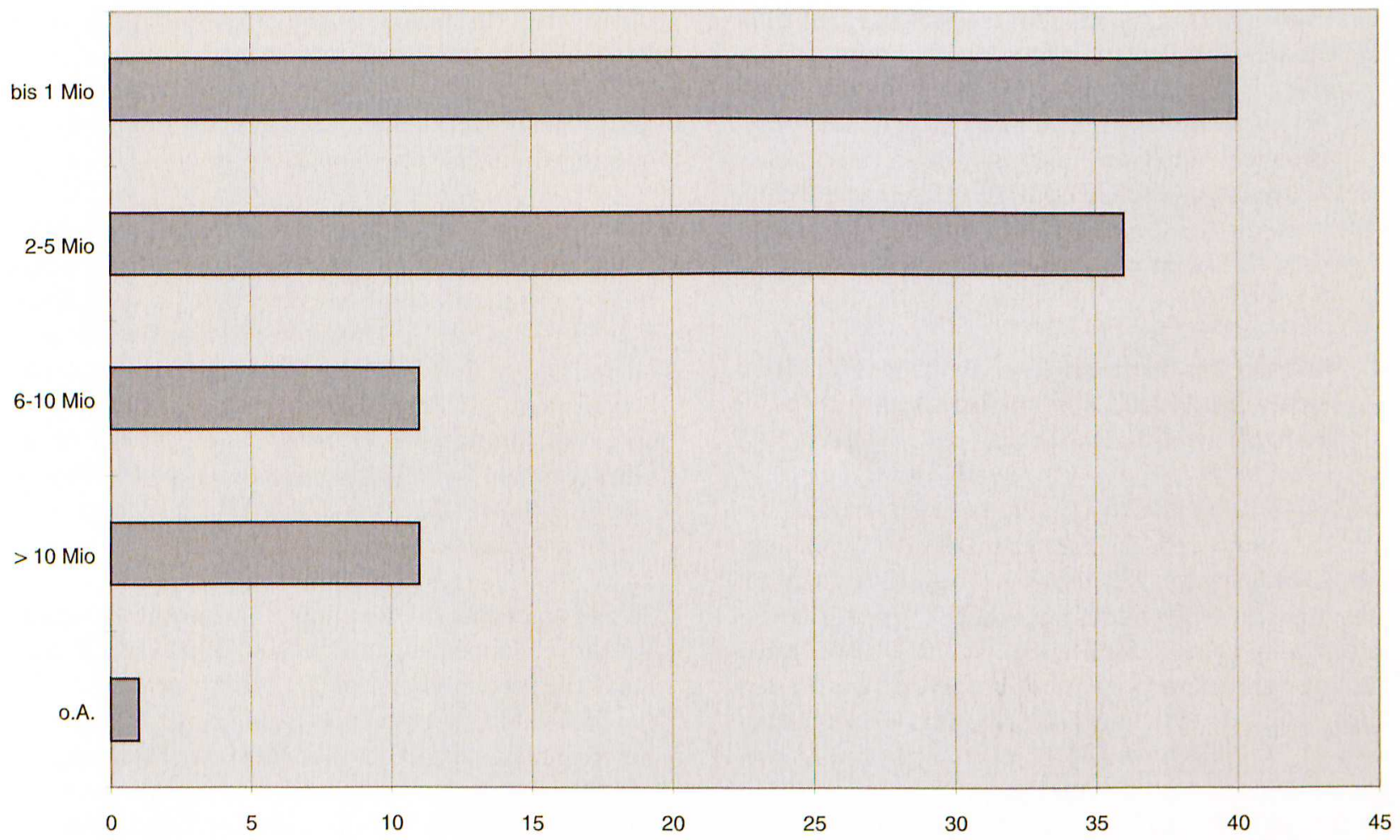

Abb. 3: Betriebsgröße Brandenburger Unternehmen (Umsatz) ( $n=70$, Angaben in \%) 


\subsection{Entwicklungsprobleme und -potentiale der befragten Unternehmen}

\section{Forschungsintensität}

Für Chancen und Wettbewerbsfähigkeit von Unternehmen auf dem Gebiet Technische Lebenshilfen stellt die Entwicklung und intensive Nutzung von modernem wissenschaftlichen und technologischen know-how eine entscheidende Voraussetzung dar. Dies erfordert eine hohe Forschungsintensität einschließlich eigener Forschungspotentiale. Deutschlandweit verfuigen 94 Prozent der befragten Unternehmen auf diesem Gebiet über eigene FuE-Potentiale. Für Südbrandenburg betrifft dies immerhin auch 61 Prozent der erfaßten Unternehmen. Die Unternehmen sind sich durchaus der notwendigen zusätzlichen Anstrengungen bewußt, denn 38 Prozent meinen, im Fall verstärkten Engagements auf diesem Gebiet das FuE-Potential erweitern bzw. neu aufbauen zu müssen.

Neben den typischen Informationsdefiziten haben sich die Unternehmen mangels eigener Erfahrungen und Ressourcen mit zusätzlichen Problemen auseinanderzusetzen, für deren Lösung ein zu schaffendes Transferzentrum Unterstuitzung bieten könnte. Neben der Erhöhung der Transparenz der Nachfrage sowie der Kaufkraft der verschiedenen Gruppen älterer Menschen durch Informationen, Demonstrationen, Produkttests und andere Maßnahmen muißte es deshalb wesentliche Beiträge dazu leisten, die Entwicklung marktfähiger, innovativer Leistungsangebote durch verstärktes kooperatives bzw. vernetztes Agieren der Unternehmen mit Forschungseinrichtungen und den Abnehmern der Produkte zu unterstuitzen.

\section{Marktorientierung und Kundennähe}

Ein großer Anteil der auf der Basis gesundheitspolitischer Regulierungen versorgten Technischen Hilfen wird nicht direkt an die eigentlichen Kunden geliefert (beeinträchtigte Senioren, Behinderte, Rehabilitanten, Kliniken, Pflegedienste, Sozialstationen u.a.). In der bundesweiten Befragung betrug dieser Anteil 43 Prozent. Die Hauptmittler in der Kette zwischen Hersteller und Kunden sind Ärzte, Beratungsstellen und die Sanitätsfachhäuser, an die 30 Prozent aller Lieferungen erfolgen. Sozialgesetzgebung und Krankenkassen spielen in diesem Prozeß ebenfalls eine nicht unerhebliche Rolle. Es besteht also oft kein direkter Kontakt zwischen Anbietern und Kunden. Dieses Problem der mangelnden Kundennähe könnte auch für Brandenburger Unternehmen von Relevanz sein, denn nur 42 Prozent der hier befragten Unternehmen liefern direkt an den privaten Endverbraucher - allerdings bezogen auf ihr gesamtes Leistungsangebot und nicht nur auf den Bereich Technische Hilfen. Die Mehrheit (58 Prozent) ist als Zulieferer für andere Unternehmen tätig.

Die Brandenburger Unternehmen sind nicht nur was ihre Märkte anbelangt (vgl. Abb. 4), sondern auch hinsichtlich der erforderlichen Zulieferungen für ihre Leistungsangebote mehrheitlich überregional orientiert. Mehr als 85 Prozent beziehen diese aus Deutschland, immerhin 55 Prozent aus dem Ausland, 31 Prozent aus Brandenburg und nur 26 Prozent nutzen regionale bzw. 23 Prozent lokale Zulieferungen.

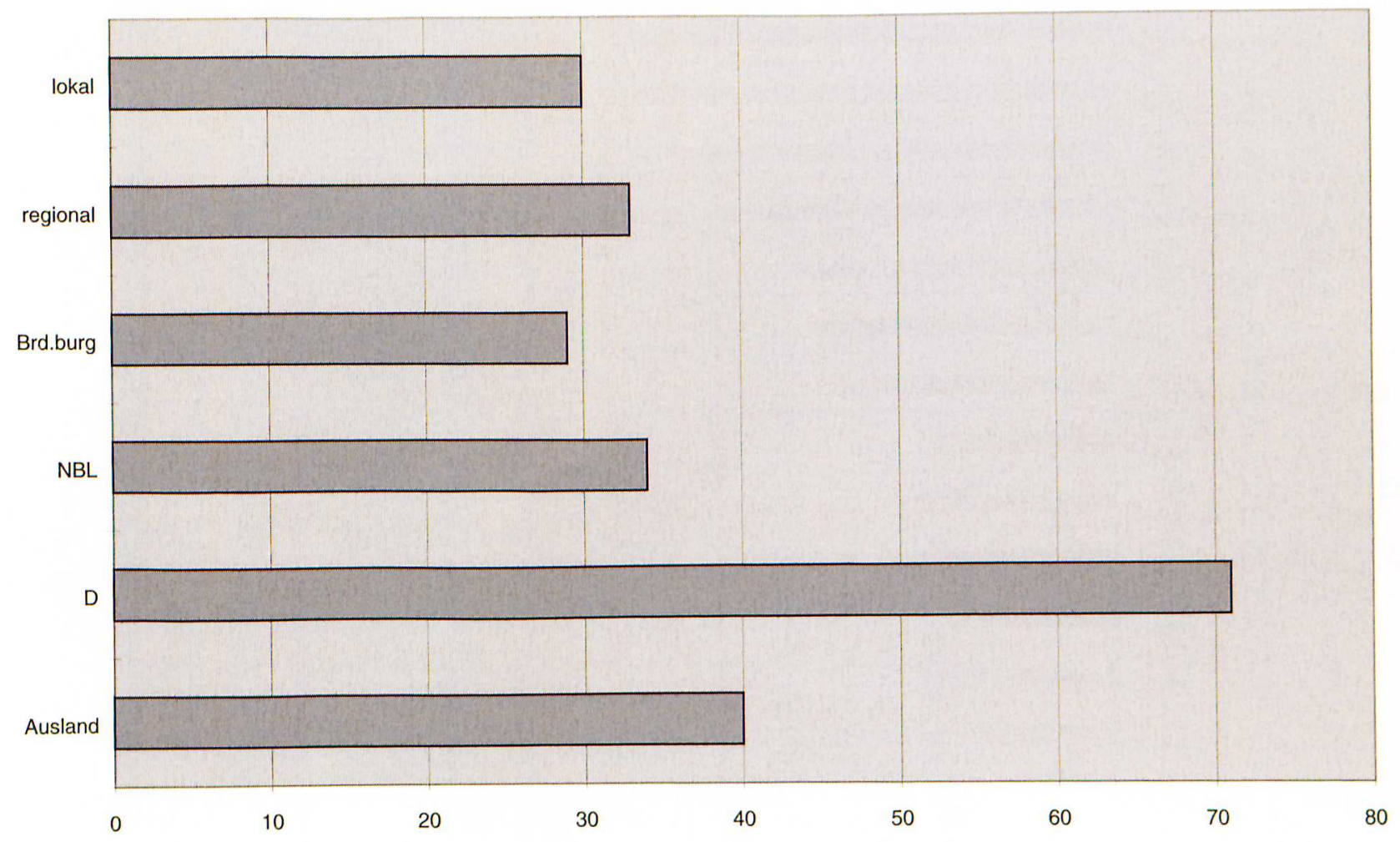

Abb. 4: Absatzmärkte Brandenburger Unternehmen ( $n=70$, Angaben in \%) Mehrfachnennungen zugelassen 


\section{Kooperationsverhalten}

Ungeachtet dessen, ob die befragten Unternehmen Endhersteller oder Zulieferer sind, ist ihr Kooperationsverhalten, ähnlich wie schon aus der bundesweiten Befragung deutlich wurde, fast ausschließlich vertikal orientiert. Das betrifft sowohl die FuE-Kooperationen als auch die Leistungserstellung selbst. Horizontale Kooperationen, die eine Chance zur effizienten Erstellung von Systemlösungen und Komplettangeboten für bestimmte Funktionsbereiche Technischer Hilfen bzw. Alltagstechnik und Wohnungsanpassung sein können, fehlen nahezu völlig. Dies wird von den Firmen selbst auch als defizitär empfunden. Ausgehend von der Tatsache, daß man bezogen auf die genannten Leistungsangebote der befragten Firmen kaum von starker Konkurrenz innerhalb der Region sprechen kann, da sie in den jeweiligen Gebieten wie z.B. Medizintechnik oder Wohnungsanpassung Spezialleistungen anbieten, sind die Voraussetzungen für eine verstärkte horizontale Kooperation relativ günstig.

\subsection{Offensive Firmenstrategien mit Synergiepotentialen}

Die Bereitschaft der befragten Firmen, sich erstmalig oder verstärkt auf Geschäftsfeldern im Bereich Technische Lebenshilfen zu engagieren, ist Ausdruck für eine offensive Geschäftsfeldstrategie und ein entsprechendes Entwicklungspotential auf der Anbieterseite in der Region.
In der bisherigen Unternehmensorientierung uiberwiegen Leistungsangebote fuir folgende Funktionsbereiche Technischer Hilfen: Überwachung/Sicherheit, Notrufsysteme, Mobilität, Spezialmöbel, Kommunikation, Halten/Stiitzen, Liegen/Sitzen, Therapie, Pflege, Lernen/ Spielen.

Angesichts des erforderlichen Know-hows, der Forschungsintensität und der notwendigen Ressourcen sollten Neueinsteiger in das Gebiet sich auf solche Leistungen konzentrieren, die nahe an ihren Kernkompetenzen hinsichtlich der technologischen Wissensbasis, der Fertigungstechnik sowie ihrer Vertriebserfahrungen und Marketingkonzepte liegen, um über entsprechende Synergiepotentiale ihre Wettbewerbsfähigkeit zu sichern. Für die genannten Bereiche werden von den Unternehmen überwiegend hohe Synergien zu ihren derzeitigen Kernkompetenzen gesehen (vgl. Abb. 5).

Gleichzeitig sind sich die Unternehmen dessen bewußt, daß sie bei einem verstärkten Engagement auf dem Gebiet Technische Lebenshilfen selbst weitere unternehmensinterne Voraussetzungen schaffen müssen. So wïrden 38 Prozent der Unternehmen im Falle der Geschäftsfelderweiterung neue Mitarbeiter einstellen und 41 Prozent geben an, ihre Marketingkonzepte uiberarbeiten zu müssen. Die Notwendigkeit zusätzlicher Investitionen in die Fertigungstechnik sehen derzeit nur 17 Prozent der befragten Unternehmen, allerdings schätzen 48 Prozent ein, daß sie zusätzliches Fremdkapital benötigen, um ihre offensiven Strategien umzusetzen.

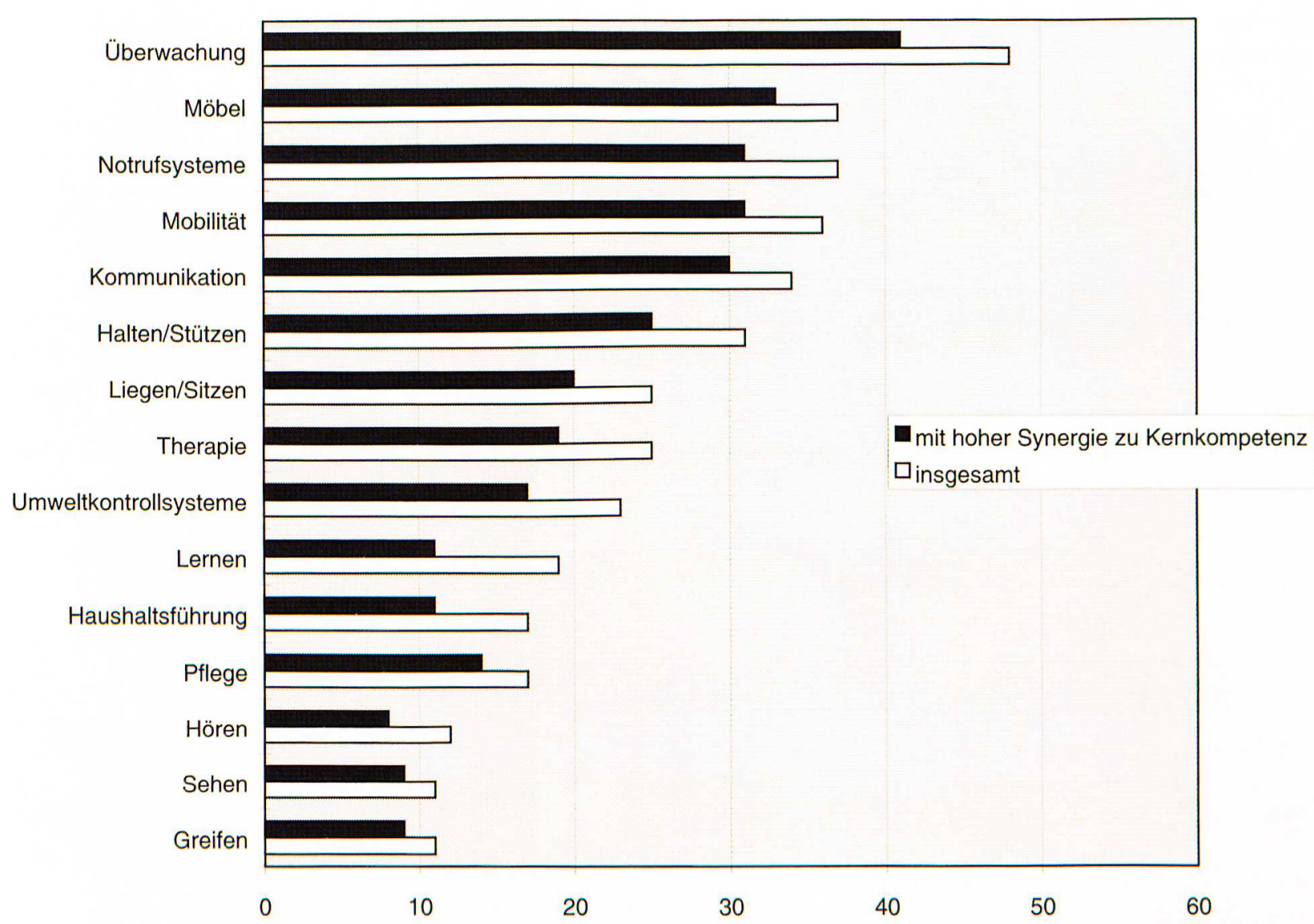

Abb. 5: Bereiche Technischer Hilfen im unternehmerischen Interesse ( $n=70$, Angaben in \%) Mehrfachnennungen zugelassen 
Unter innovationsstrategischem Gesichtspunkt ist die hohe Bereitschaft von über 66 Prozent der Firmen hervorzuheben, gewissermaßen von außen angebotene wissenschaftlich-technische Problemlösungen in ein vermarktbares Leistungsangebot umzusetzen. Dies bietet einen Ansatz zur vertieften Kooperation mit wissenschaftlichen Einrichtungen, die solche entwicklungsfähigen Lösungsprinzipien erarbeiten. Auf dem Unternehmerworkshop wurden aber auch die Voraussetzungen genannt, die aus Sicht der Firmen erforderlich sind, um dies auch tatsächlich realisieren zu können. Zu diesen Voraussetzungen zählen z.B. die Paßfähigkeit der Produktidee zur Geschäftsfeldstrategie, die Finanzierbarkeit solcher Transferprojekte und das Vorhandensein wirtschaftlicher Eckdaten für eine kostengünstige Leistungserstellung (z.B. Losgrößen, Kosten, Preise).

\subsection{Zutrittschancen für Unternehmen}

Die hochgradige Differenzierung der Märkte, die unterschiedlichen Wirkungen der nachfragebeeinflussenden Faktoren auf die verschiedenen Marktsegmente in diesem Bereich sowie die Inhomogenität der Gruppe „ältere Menschen" und die oft nur über mehrere Stufen vermittelte Kundennähe der Firmen erschweren die Erstellung von Leistungsangeboten, die den Anforderungen der Kunden entsprechen und deren Kaufverhalten verstärken.

Neu hinzutretende oder sich in diesem Feld verstärkt engagierende Unternehmen haben vor allem dann eine Chance, sich am Aufbau entsprechender Marktpotentiale erfolgreich zu beteiligen, wenn es ihnen gelingt

- bestimmte Problemsituationen älterer Menschen fruiher, schneller, gründlicher als Konkurrenten zu erkennen und zu analysieren,

- dafür spezifische wettbewerbsfähige Lösungen anzubieten, um aus Beduirfnissen Nachfrage zu entwickeln und

- mit konkreten Leistungsangeboten und Vermarktungsstrategien (Marketinginstrumenten) dem starken Einfluß individueller Faktoren auf das Verhalten $\ddot{A}$ lterer zu Technischen Lebenshilfen Rechnung zu tragen.

Insgesamt kann eingeschätzt werden, daß sich die Zutrittschancen für Unternehmen auf dem Gebiet „Gesundheitstechnik - Technische Lebenshilfen, altersgerechtes Wohnen“ in Brandenburg nicht wesentlich von denen unterscheiden, die für Gesamtdeutschland gelten. Die bundesweite Befragung hat ergeben, daß in größeren Perioden von vor 1950 bis in die Gegenwart Unternehmen neu auf diese Märkte hinzugetreten sind. Diese Tendenz hat sich verstärkt, so kamen von 1970 bis 1979 ca. 13 Prozent der befragten Unternehmen, von 1980 bis 1989 ca. 44 Prozent und von 1990 bis 1994 nochmals ca. 28 Prozent hinzu.

Mit der öffentlichen Förderung von Forschungen auf dem Gebiet Gesundheitstechnik - Technische Lebenshilfen, altersgerechtes Wohnen in Deutschland, aber auch durch die Europäische Union sind derzeit gute Voraussetzungen dafür gegeben, daß Unternehmen den erforderlichen Zugang zu neuem wissenschaftlichen und technologischen Know-how erhalten können. So fördert beispielsweise gegenwärtig die Deutsche Forschungsgemeinschaft (DFG) mit einem interdisziplinären Forschungsprojekt „sentha“ (Seniorengerechte Technik im häuslichen Alltag), das durch das Zentrum für Technik und Gesellschaft an der Technischen Universität Berlin koordiniert wird und an dem auch die Technische Universität Cottbus beteiligt ist, zunächst fuir drei Jahre Forschungen zur seniorengerechten Kommunikations- und Sicherheitstechnik sowie zum Design von Produkten, zur Konstruktionstechnik, zur Technikbewertung und zur Architektur. Weitere Forschungen auf diesem Gebiet werden an der Fachhochschule Lausitz, an der Fachhochschule Potsdam und an der Universität Potsdam durchgefuihrt.

Für Südbrandenburg besteht die einmalige Chance, das unternehmerische Potential frühzeitig an diese Forschungsaktivitäten heranzuführen und gewissermaßen aus erster Hand an neuen Forschungs- und Entwicklungsergebnissen für den Markt „Alltagstechnik für ältere Menschen“ zu partizipieren.

\section{Ausblick}

Begründet durch die Machbarkeitsstudie hat die Stadt Senftenberg einen Antrag gestellt, mit Hilfe von Fördermitteln der EU und des Landes Brandenburg ein Transferzentrum „Gesundheitstechnik, Technische Lebenshilfen, altersgerechtes Wohnen“ einzurichten. Damit würde eine Einrichtung geschaffen, die dazu beitragen kann, Probleme an den Schnittstellen zwischen Nachfragern (Senioren, beeinträchtigte und behinderte Menschen), leistungsvermittelnden Sozialeinrichtungen, Unternehmen und Forschungseinrichtungen lösen zu helfen und damit vorhandene Defizite im Prozeß der Leistungserstellung und Vermarktung im Bereich seniorengerechte Technik zu überwinden und Marktpotentiale zu erschließen. Solche Entwicklungen könnten den Strukturwandel in der Wirtschaft des Landes Brandenburg maßgeblich beeinflussen.

Zur Unterstiitzung der zukünftigen Tätigkeit des Transferzentrums sowie vor allem der Unternehmen bei der Überwindung vorhandener Schwächen, z.B. im Innovationsmanagement, bei der Marktforschung und der Lösung konkreter Marketingprobleme, haben die Autoren ein neues Projekt im Rahmen des Programms „Förderung anwendungsorientierter Forschung und Entwicklung an Fachhochschulen" beim BMBF eingereicht. 


\section{Literatur}

[1] vgl.: Brandt, M.; Voß, R.: Technische Lebenshilfen im Alter - Ein Zukunftsmarkt für mittelständische Unternehmen mit wirtschaftsstrukturellen Effekten in Südbrandenburg? Teilprojekt zum Transferprojekt „Gesundheitstechnik - Technische Lebenshilfen, altersgerechtes Wohnen“. Berlin 1998. 76 S.

[2] vgl.: Ministerium für Wirtschaft, Mittelstand und Technologie des Landes Brandenburg (Hrsg.): Brandenburg auf dem Weg in die Zukunft. Technologiekonzeption des Landes Brandenburg. Potsdam 1994

[3] vgl.: Große, U.; Hartmann, F.; Voß, R. unter Mitarbeit Brandt, M.: Internationale Trends in der Biotechnologie. Auswertung aktueller Trends in biotechnologischen Innovationslinien mit Relevanz für kleine und mittlere Unternehmen. Berlin 1998

[4] vgl.: Ministerium für Wirtschaft, Mittelstand und Technologie des Landes Brandenburg (Hrsg.): Sechstes Industriesymposium Brandenburg. Marktzugang durch „Konsolidierung im Verbund“. Potsdam 1998

[5] Reents, H. (Hrsg.): Handbuch der Gerontotechnik. Landsberg/Lech 1996

[6] Die Gemeinschaftsinitiative RECHAR II der Europäischen Kommission zielt auf die Verbesserung des Arbeitsmarktes in den Regionen, die vom Strukturwandel im Bereich Kohlebergbau in Mitleidenschaft gezogen wurden. Das Land Brandenburg beteiligt sich mit einer entsprechenden Kofinanzierung an dieser Fördermaßnahme.

[7] vgl.: BBJ Servis GmbH u.a.: Voraussetzungen und Bedingungen für die Schaffung des Transferzentrums Gesundheitstechnik,Technische Lebenshilfen, altersgerechtes Wohnen". Machbarkeitsstudie im Auftrag der Technologie- und Innovationsagentur (T.IN.A.) Brandenburg GmbH. Cottbus 1998

[8] vgl: Mollenkopf, H.: Technik zur Integration älterer Menschen. In: Mollenkopf, H.; Hampel, J.; Zapf, W.: Technik, Alter, Lebensqualität. Stuttgart 1994

[9] vgl.: ebenda

[10] vgl.: McEvoy, C.: Technische Hilfen im Alter. In: Reents, $\mathrm{H}$. (Hrsg.): Handbuch der Gerontotechnik. Landsberg/Lech 1996, 1. Erg.Lfg. 12/97, S. 1

[11] vgl.: Ministerium für Arbeit, Soziales, Gesundheit und Frauen (Hrsg.): Landesaltenbericht. Altenpolitik im Land Brandenburg. Potsdam 1998

[12] vgl.: Lohrum, M.: Barrierefreiheit und Benutzerfreundlichkeit. Neue Anforderungen an die Unternehmen in einer sich wandelnden Gesellschaft. In: Gerontotechnik 1/97. S. 8-9

[13| vgl.: Einkommen im Alter: Wer bekommt wie viel? In: proAlter: 30(3)1997, S. 6-11

[14] vgl.: Die Alten der Zukunft - Bevölkerungsstatistische Datenanalyse. Schriftenreihe des Bundesministeriums für Familie und Senioren. Band 32, S. 104

[15] vgl.: proAlter 2/1996, S. 36

[16] vgl.: Bundesarbeitsgemeinschaft Wohnungsanpassung e.V. Verein zur Förderung selbständigen Wohnens älterer und behinderter Menschen: Informationsmaterial. München 1994

[17] vgl.: Ministerium für Arbeit, Soziales, Gesundheit und Frauen (Hrsg.): Landesaltenbericht. a.a.O.

[18] vgl.: Wohnen im Alter: Studie zur Wohn- und Lebenssituation älterer Bürger in Zeuthen. Wildau/Zeuthen 1997
[19] vgl.: Mollenkopf, H.; Flaschenträger, P.: Mobilität im Alter. In: Reents, H. (Hrsg.): Handbuch der Gerontotechnik. 1. Erg.Lfg. 12/97, S. 1

[20] vgl.: Förderverein für Gerontotechnik (Hrsg.): GerontoTechnik, Heft 4/1997, S. 11

[21] vgl.: Ministerium fuir Arbeit, Soziales, Gesundheit und Frauen (Hrsg.): Analysen zur Lebenssituation älterer Menschen und zu ihrer Betreuung und Versorgung im Land Brandenburg. Potsdam 1994

[22] vgl.: Kliemke, Ch.; Marfels, G.; Böttcher, E.: Gründung eines Zentrums fuir Technische Lebenshilfen (Hilfsmittelzentrum) in Berlin. TU Berlin, Institut für Krankenhausbau. Mai 1990

[23] vgl.: von Bandemer, St.; Born, A.; Bußmann, U.; Scharfenorth, K.: Technik und Dienstleistung für mehr Lebensqualität im Alter. Gelsenkirchen 1995

\section{Autoren}

\section{Prof. Dr. phil. Rainer Voß}

Technische Fachhochschule Wildau, Leiter des Instituts für Regionale Innovationsforschung Bahnhofstraße

15745 Wildau

Tel. (0 33 75) 508-235

Fax (0 33 75) 508-214

E-Mail: rvoss@igw.tfh-wildau.de

\section{Dr. oec. Martina Brandt}

Mitarbeiterin im Institut für Regionale Innovationsforschung

Prenzlauer Promenade 149-152

13189 Berlin

Tel. (0 30) 4797278

Fax (0 30) 4797259

E-Mail: iriinstitut@aol.com 